\title{
Effects of plant protection UAV-based spraying on the vertical distribution of droplet deposition on Japonica rice plants in Northeast China
}

\author{
Yingli $\mathrm{Cao}^{1,2,3}$, Fenghua $\mathrm{Yu}^{1,2,3}$, Tongyu $\mathrm{Xu}^{1,2^{*}}$, Wen $\mathrm{Du}^{1,2}$, Zhonghui Guo ${ }^{1}$, Haiyan Zhang ${ }^{1}$ \\ (1. College of Information and Electrical Engineering, Shenyang Agricultural University, Shenyang 110866, China; \\ 2. Liaoning Engineering Research Center for Information Technology in Agriculture, Shenyang 110866, China; \\ 3. State Key Laboratory of Robotics, Shenyang Institute of Automation, Chinese Academy of Sciences, Shenyang 110016, China)
}

\begin{abstract}
In order to study the regularity of the vertical distribution of droplet deposition on rice plants during pesticide spraying operated by a low-flying multi-rotor plant protection unmanned aviation vehicle (UAV), water-sensitive spray cards were placed on the leaves at the top, in the middle, and at the bottom of rice plants to acquire data on droplet deposition. In this study, a suspension containing tricylazole and hexaconazole was used in the spraying. The water-sensitive spray cards were analyzed by the droplet deposition processing software iDAS to obtain the vertical distribution of the droplets sprayed by the plant protection UAV. The results showed that 1) significant variation was found in the coverage of the droplets in different vertical positions of the rice plants. Within the effective spray width, the best coverage of the droplets was found in the area just below the rotors, whereas the coverage of areas farther away from the rotors was poor. For the different vertical positions of the rice plants, the overall droplet coverage was $58.38 \%$ at the top, $33.55 \%$ in the middle, and $11.34 \%$ at the bottom of the plants; 2) for all vertical positions, the average size of the droplets ranged between $110 \mu \mathrm{m}$ and $140 \mu \mathrm{m}$, which was suitable for the control of plant diseases and insect pests. The highest droplet density was found at the top of the plants, and the distribution of the droplet density was similar in the middle and at the bottom of the rice plants; 3 ) the diffusion ratio at the top of the rice plants $(0.84)$ was better than that in the middle $(0.57)$ and at the bottom $(0.37)$. The overall relative span could meet the requirements for the actual application. Except for the position in the middle of the plants, the relative span for the other positions of the plants was over 0.67 , which is the standard value. This study demonstrated the distribution regularity of droplet deposition along with the vertical direction of rice plants during UAV-based spraying, which is of guiding significance for the use of UAVs in plant protection, the improvement of chemical utilization efficiency, and the reduction of pesticide and fertilizer pollution.
\end{abstract}

Keywords: plant protection UAV, droplet deposition, Japonica rice, droplet size, vertical distribution, spraying, Northeast China

DOI: $10.25165 /$ j.ijabe.20211405.5490

Citation: Cao Y L, Yu F H, Xu T Y, Du W, Guo Z H, Zhang H Y. Effects of plant protection UAV-based spraying on the vertical distribution of droplet deposition on Japonica rice plants in Northeast China. Int J Agric \& Biol Eng, 2021; 14(5): $27-34$.

\section{Introduction}

Rice is a staple food crop grown in 39 countries and consumed by 2.7 billion Asians ${ }^{[1]}$. Rice grown in Northeast China is mainly Japonica rice. Originated in China, Japonica rice is characterized by short stems, narrow leaves, short and thick grains, a high milling yield, and a low volume expansion ratio. Rice production is directly affected by plant diseases. Rice diseases are common in rice fields in Northeast China; in general, these diseases result in yield losses of $10 \%-20 \%$ and up to $40 \%-50 \%$ in severe cases. On occasion, even $100 \%$ yield loss can occur, posing a serious threat to the high and

Received date: 2019-10-29 Accepted date: 2021-06-08

Biographies: Yingli Cao, $\mathrm{PhD}$, Professor, research interest: agricultural remote sensing, Email: caoyingli@syau.edu.cn; Fenghua Yu, PhD, research interest: agricultural remote sensing, Email: adan@syau.edu.cn; Wen Du, PhD, research interest: agricultural remote sensing, Email: duwen@syau.edu.cn; Zhonghui Guo $\mathrm{PhD}$ candidate, research interest: agricultural remote sensing, Email: 489336068@qq.com; Haiyan Zhang, PhD candidate, research interest: agricultural remote sensing, Email: 1322366847@qq.com.

*Corresponding author: Tongyu $\mathbf{X u}, \mathrm{PhD}$, Professor, research interest: agricultural remote sensing. College of Information and Electrical Engineering, Shenyang Agricultural University, Shenyang 110866, China. Tel: +86-13889270306, Email: yatongmu@163.com. stable yield of Japonica rice ${ }^{[2]}$. At present, chemical control is the main measure for the prevention and control of rice diseases. Manual spraying is the primary method for the prevention and control of rice blast in most areas of China. However, it is very difficult to walk in paddy fields, therefore manual spraying requires a high degree of labor intensity. For traditional manual spraying, approximately $20 \%$ to $30 \%$ of the tiny droplets drift to non-target areas due to the flow of air $^{[3]}$. The use of excessive amounts of chemicals is not only wasteful but also harmful to the environment and the spraying operators. In addition, the traditional method of chemical application cannot prevent the outbreak of rice blast in a timely and rapid manner. Therefore, it is particularly important to prevent the occurrence of Japonica rice diseases by increasing the mechanization level.

The utilization of unmanned aviation vehicles (UAVs) for chemical applications has great potential in modern agriculture in China. Despite the rapid development of plant protection UAVs, they are utilized in less than $3 \%$ of the cultivated areas in China. Thus, in terms of agricultural aviation, China lags far behind the United States, Russia, Australia, Canada, Brazil, Japan, and South Korea. In the sparsely populated areas of the United States, manned fixed-wing aircraft is the most popular form of agricultural aviation $^{[4-7]}$. The development mode of agricultural aviation in 
Russia, Australia, Canada, and Brazil is the same as that in the United States, which is characterized by the use of manned fixed-wing aircraft and rotary-wing helicopters ${ }^{[8]}$. In contrast, Japan and South Korea have smaller fields and more hills and mountains, which are not suitable for manned fixed-wing aircraft. Therefore, agricultural aviation in these areas is dominated by small UAVs that are also called unmanned aerial vehicles ${ }^{[9-11]}$. The lands are mainly plains in Northeast China, and therefore multi-rotor UAVs are suitable for spraying above the terrain ${ }^{[12,13]}$. UAVs do not need airports and have the advantages of low flying height, low weight, flexible movement, and high efficiency in spraying ${ }^{[14,15]}$.

Liaoning Province is an important grain-producing area in China where rice is one of the main cultivated crops. In recent years, the increased use of agricultural chemicals caused soil degradation, environmental pollution, and rice quality downgrading. In 2015, the Ministry of Agriculture and Rural Affairs of the People' formulated the "Plan for Achieving Zero Growth of the Amount of Agricultural Chemical Use by 2020." The "13th Five-Year Plan" listed the effective operation and management of rice plant protection as one of the key means to improving the efficiency of rice production and reducing the amount of agricultural chemical use ${ }^{[16]}$. Because multi-rotor plant protection UAVs are small in size, do not need special runways, and can be operated easily and safely, they have been developed rapidly and used in regional plant protection in recent years. $\mathrm{Ru}$ et al. ${ }^{[2]}$ designed a UAV-based small volume spraying system with remote control, tested its performance for centrifugal spraying, and explored related theoretical issues. Jorge et al. ${ }^{[1]}$ developed a novel and practical application system capable of being was mounted on an unmanned aerial vehicle for agrochemical spraying task, tested the quality of application in olive and citrus orchards and compared with those of conventional treatment. Du et al. ${ }^{[12]}$ used a multi-rotor UAV to spray foliar fertilizer at the rice filling stage and analyzed the effect of droplet deposition on the top leaves of rice plants. Berner et al. ${ }^{[18]}$ tested the influence of air stream produced by UAV on the deposition of the sprayed liquid. The test results showed that the air stream from a flying UAV was not only exerted an influence on the distribution of the volume of sprayed liquid but also influenced the change in concentrations of the biological plant protection agent. Zhang et al. ${ }^{[13]}$ investigated the efficiency of UAV-based spraying in controlling rice grasshoppers. As compared with the traditional land-based application of agricultural chemicals, the spraying efficiency was increased by over 60 times by using UAVs. Furthermore, the amount of pesticide use was reduced by $20 \%-30 \%$ and the labor intensity was greatly reduced. The use of UAVs provides assurance for the effective prevention and control of rapid outbreaks of rice blasts. Teske et al. ${ }^{[19]}$ developed a new retention model to predict the drift and deposition of sprays released from UAVs based on AGDISP and CHARM models, explored the theoretical behavior of two UAVs and compared the prediction results of the new model with that of AGDISP. The results showed that the new model was more suitable to predict the performance of UAVs than AGDISP. And new model pinpointed the importance of a critical flight speed. Yuan et al. ${ }^{[16]}$ elucidated the relation of droplet size and coverage density with the effects of pesticides, fungicides, and herbicides, which provided a reference for the precise application of chemicals using UAVs and guidance for agricultural production. Chen et al. ${ }^{[20]}$ studied the effects of combinations of different flight and spray parameters on the droplet distribution on the leaves at the top of rice plants, as well as the influence of wind field on spraying, which is of great guiding significance for the rational use of UAVs in plant protection and the improvement of spraying efficiency.
The traditional chemical application for rice plant protection mainly includes manual spraying, ground machinery application, and fixed-wing aircraft operation, among which manual spraying has the shortcomings of great labor intensity, high risk, low work efficiency, and long application duration ${ }^{[21,22]}$. It is difficult for ground machinery to move in the paddy field and ground machines are prone to damaging the field and rice plants. Fixed-wing aircraft application is constrained by site conditions, and the spray drift is more serious and costly than other methods. As a newly developed technology in the field of plant protection, plant protection UAVs can effectively overcome the shortcomings of traditional plant protection technology; they are becoming the first choice in rice plant protection At present, the load of plant protection UAVs is very limited. The capacity of their chemical tank is usually $5-20 \mathrm{~kg}$. Therefore, ultra-low-volume spraying technology is needed to apply chemicals using UAVs ${ }^{[23,24]}$

This study was designed with the primary aim of improving the current state of plant protection UAVs used in rice production in Liaoning Province, China. By using a multi-rotor plant protection UAV in chemical application, this study investigated the effects of different flight parameters on the distribution of droplet deposition on rice plants. This study also identified the regularities of the vertical distribution of droplet deposition on rice plants and provided data and a theoretical basis for increasing the efficacy of chemical application by plant protection UAVs to ultimately reduce the amount of chemical use.

\section{Materials and methods}

In this study, a suspension of $27 \%$ fungicide containing $22.5 \%$ tricyclazole and $4.5 \%$ hexaconazole was used to prevent rice neck blast. The Japonica rice variety was Jindao 106. Row spacing was $30 \mathrm{~cm}$ and the space between plants in a row was $18 \mathrm{~cm}$. At the time of spraying, the crop was at the heading stage and the average plant height was $0.75 \mathrm{~m}$.

\subsection{Plant protection UAV}

The UAV used in this study was the MG-1P eight-rotor electric unmanned helicopter for plant protection (DJI Innovation Technology Co., Ltd., Shenzhen City, Guangdong Province, China), which is shown in Figure 1. The general performance indexes of this UAV are listed in Table 1.

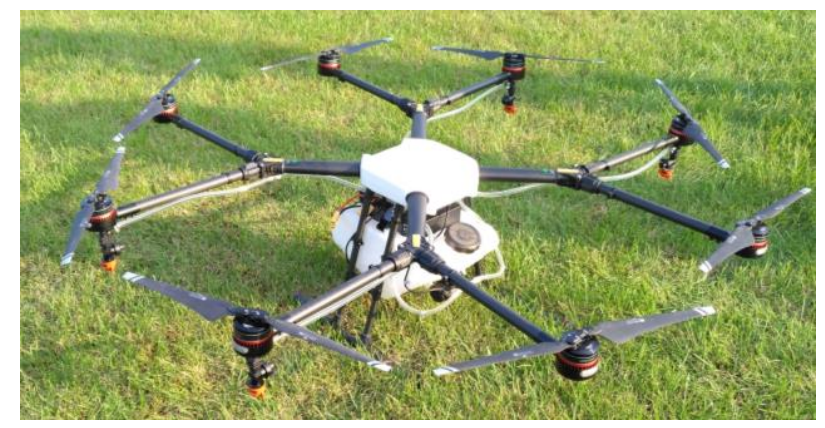

Figure 1 Plant protection unmanned aviation vehicle

Table 1 General performance indexes of MG-1

\begin{tabular}{lc}
\hline \multicolumn{1}{c}{ General parameter } & Value or norm \\
\hline Type & MG-1 Eight-rotor electric unmanned aviation vehicle \\
Size $/ \mathrm{mm} \times \mathrm{mm} \times \mathrm{mm}$ & $1471 \times 1471 \times 482$ \\
Diagonal wheelbase $/ \mathrm{mm}$ & 1520 \\
Flight $\mathrm{speed} / \mathrm{m} \cdot \mathrm{s}^{-1}$ & $0-8$ \\
Spraying width $/ \mathrm{m}$ & $4-6$ \\
\hline
\end{tabular}


The UAV-based liquid chemical spraying system consisted of an anti-shaking chemical tank, a water pump, chemical conducting tubing, and ceramic nozzles. The spraying device had four centrifugal nozzles, which were located below the body of the UAV and pointed vertically downward. The flow rate of each nozzle was $0.43 \mathrm{~L} / \mathrm{min}$. The environmental information during spraying was obtained by using a portable anemometer and a handheld temperature and humidity detector. Water-sensitive spray cards produced by Syngenta (Switzerland) were used to acquire information related to distribution, size, and coverage of the droplets sprayed by the UAV.

\subsection{Methods}

\subsubsection{Site and materials}

The trial was performed on July 19, 2018, in Dawa District, Panjin City, Liaoning Province, China. The rice was at the heading stage and the average plant height was $115 \mathrm{~cm}$. The rice variety was Jindao 106. During spraying, the average ambient temperature was $25.75^{\circ} \mathrm{C}$, the relative humidity was $33.01 \%$, and the wind speed was $1.375 \mathrm{~m} / \mathrm{s}$.

\subsubsection{Arrangement of data collection}

A rectangular field of $202.35 \mathrm{hm}^{2}$ was selected for the trial in this study. The flying height of the UAV was $1 \mathrm{~m}$ above the top of the rice plants, and the flying speed was set at $1.5 \mathrm{~m} / \mathrm{s}$. Three replications were set for the tests. Environmental factors, such as the presence of dew on rice leaves, may produce noise on the water-sensitive spray cards. In order to reduce such noise interference on data collection, the tests started at 8:50 a.m. The water-sensitive spray cards were placed on the leaves positioned at the top, in the middle ( $60 \mathrm{~cm}$ above ground), and at the bottom of the rice plants in the vertical direction, while the UAV flew in the horizontal direction. The data collection spots were arranged along with the rows in the field, and 17 spots were distributed in each row. The length of the data collection belt was approximately $8 \mathrm{~m}$, which was larger than the spraying width; this ensured that all droplets were collected. The water-sensitive spray cards were attached to the upper and lower surfaces of the rice leaves with paperclips. During spraying, the UAV adopted the autonomous operation mode and flew along the preset route, which was the medial axis of the plot. In order to ensure accurate positioning during flight, a differential module was set in the UAV, a base station on the ground and adopted Differential Global Positioning Systems (DGPS) technology to improve the positioning accuracy. In order to avoid the error caused by the drift of the droplets, a spacing of $10 \mathrm{~m}$ was arranged between the replicates of a test, and a spacing of $15 \mathrm{~m}$ was arranged between the tests. After spraying, the water-sensitive spray cards were allowed to dry properly for $30 \mathrm{~min}$. Disposable gloves were worn to collect the water-sensitive spray cards. The water-sensitive spray cards were numbered, sealed, and brought back to the laboratory for analysis. The layout of the field plot is shown in Figure 2.

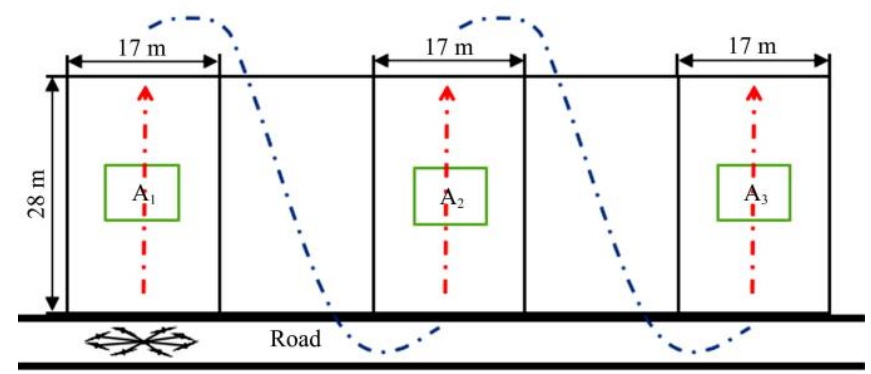

Note: $A_{1}, A_{2}$ and $A_{3}$ were three different treatments which were shown in Table 2 The width of the road was $5 \mathrm{~m}$.

Figure 2 Layout of trial field plot

Each field was $1000 \mathrm{~m}$ long and $26 \mathrm{~m}$ wide. Five rectangular field plots of $28 \mathrm{~m} \times 17 \mathrm{~m}$ were selected for five sets of different tests (Table 2).

Table 2 Treatments of the three test plot

\begin{tabular}{ccc} 
Test plot & Chemical application machinery & Chemical and dose \\
\hline $\mathrm{A}_{1}$ & Eight-rotor plant protection UAV & $\begin{array}{c}27 \% \text { Tricylazole+ } \\
5 \mathrm{~g} \text { Hexaconazole suspension }\end{array}$ \\
& & $27 \%$ Tricylazole+ \\
$\mathrm{A}_{2}$ & Eight-rotor plant protection UAV & $8 \mathrm{~g} \mathrm{Hexaconazole} \mathrm{suspension}$ \\
& & $27 \%$ Tricylazole+ \\
$\mathrm{A}_{3}$ & Eight-rotor plant protection UAV & $10 \mathrm{~g}$ Hexaconazole suspension \\
\hline
\end{tabular}

Note: UAV: unmanned aviation vehicle.

\subsubsection{Water-sensitive spray cards and reagents}

In this study, the water-sensitive spray cards used were yellow in color and sized $26 \mathrm{~mm} \times 76 \mathrm{~mm}$. Once water droplets came into contact with the card, the surface of the card quickly turned blue and the color change was distinctly visible, as shown in Figure 3.

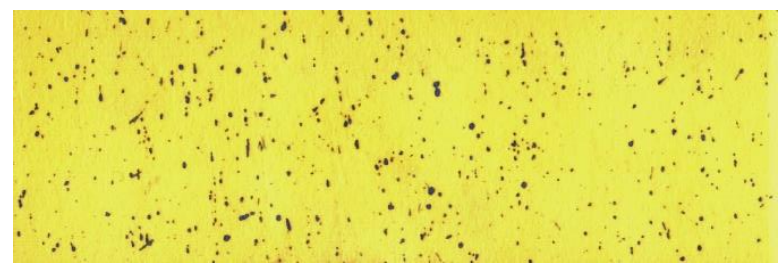

Note: The size of a water-sensitive spray card is $26 \mathrm{~mm} \times 76 \mathrm{~mm}$.

Figure 3 Water-sensitive spray card

\subsubsection{Designing of UAV operating parameters}

The purpose of this study was to determine the regularity of the distribution of droplet deposition along the vertical direction of rice plants during UAV spraying. Based on previous experience and the data in the literature ${ }^{[17,18]}$, the UAV-based spraying parameters were set as follows: the flying height was $1 \mathrm{~m}$ above the top of the rice plants, the nozzle flow rate was $0.25 \mathrm{~L} / \mathrm{min}$, and the flight speed was $1.5 \mathrm{~m} / \mathrm{s}$.

\subsection{Data processing}

After the spray cards were properly dried, the water-sensitive spray cards were numbered, individually placed into plastic bags, and brought back to the laboratory for data analysis. The cards were scanned, and the data were analyzed using image processing software iDAS to obtain the information of droplet coverage density and the amount of deposition under different flying speeds. In this study, in each position on the rice plants, the tests for droplet coverage and the number of droplets were performed three times. The Pearson correlations between the three replicates of the tests were calculated and compared, and the results are shown in Tables 3 and 4 . The results illustrated that the correlations between the replicates were all highly significant; thus, the mean of the three replicates was calculated to reduce errors.

Table 3 Correlation of coverage between the replicates of the test

\begin{tabular}{cccc}
\hline Coverage & Replicate 1 & Replicate 2 & Replicate 3 \\
\hline Replicate 1 & 1 & $0.816^{* *}$ & $0.866^{* *}$ \\
Replicate 2 & $0.816^{* *}$ & 1 & $0.843^{* *}$ \\
Replicate 3 & $0.866^{* *}$ & $0.843^{* *}$ & 1 \\
\hline Note: ${ }^{* *}$ denotes the correlation is significant at 0.01 level, the same as below.
\end{tabular}

Table 4 Correlation of droplet size between the replicates of the test

\begin{tabular}{cccc}
\hline Droplets & Replicate 1 & Replicate 2 & Replicate 3 \\
\hline Replicate 1 & 1 & $0.865^{* *}$ & $0.871^{* *}$ \\
Replicate 2 & $0.865^{* *}$ & 1 & $0.832^{* *}$ \\
Replicate 3 & $0.871^{* *}$ & $0.832^{* *}$ & 1 \\
\hline
\end{tabular}




\section{Results and analysis}

\subsection{Analysis of droplet coverage and recovery}

Figure 4 shows the coverage and recovery of droplets in different vertical positions on the rice plants. The abscissa in Figure 4 represents the actual width of each droplet collection belt, and the ordinate represents the percentage of coverage and recovery. The calculation of coverage and recovery in this study used the following two equations ${ }^{[25-27]}$ :

Coverage $=$ Soaked area/Sampling area

Recovery $=$ Amount of deposition/Sampling area

In the above equations, the soaked area refers to the cumulative area that changed color by receiving droplets on the water-sensitive spray card. The sampling area is the total area of the blank water-sensitive spray card, which is also the sum of the soaked area and the unsoaked area on the card. The recovery reflects the number of droplets deposited per unit area.

The results of droplet coverage and recovery show that during the UAV-based spraying, the distribution of the droplets on the rice leaves was uneven. The results shown in Figure 4 indicate that for UAV-based spraying with an effective spray width of $6 \mathrm{~m}$, the uniformity of the coverage was poor. In this study, the droplet coverage in the area below the nozzles was better than that in the other areas.

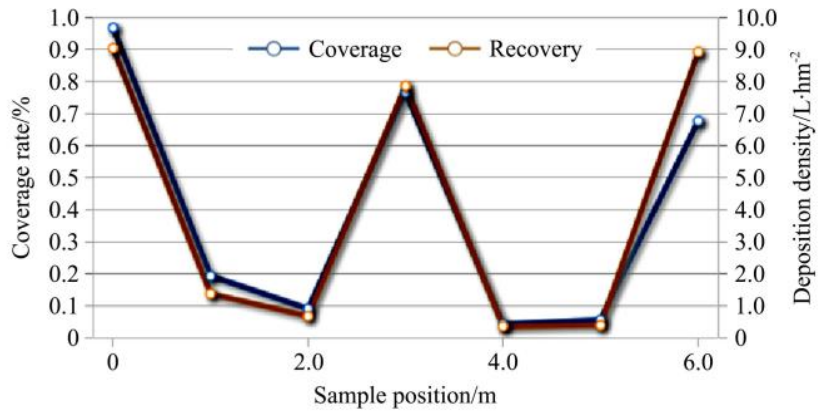

b Lower surfaces of the top leaves

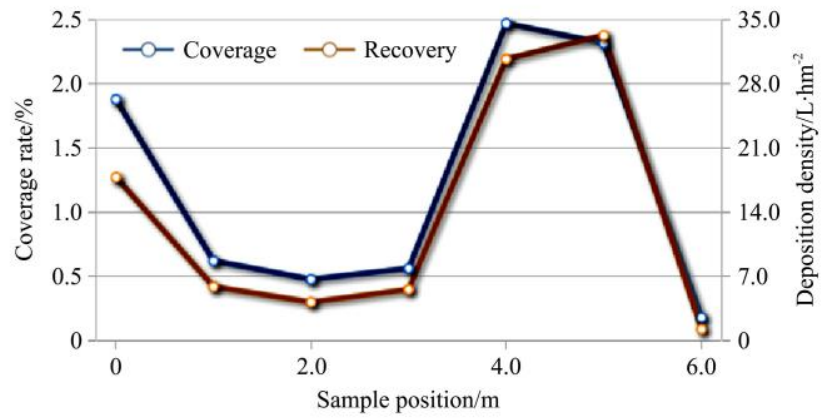

d. Lower surfaces of the middle leaves

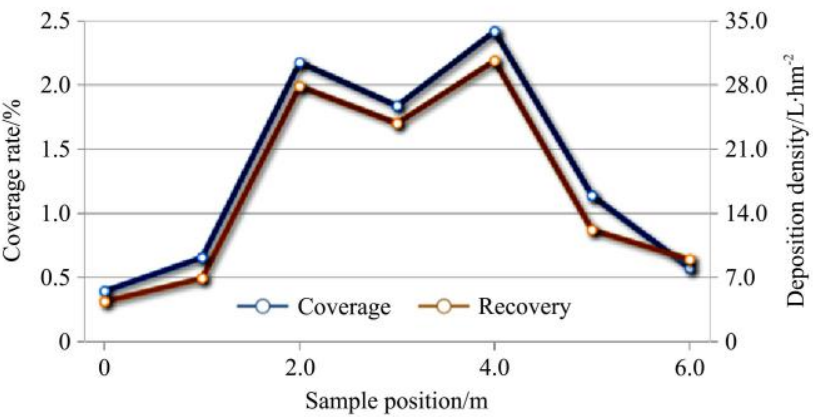

c. Upper surfaces of the middle leaves

\section{竞}
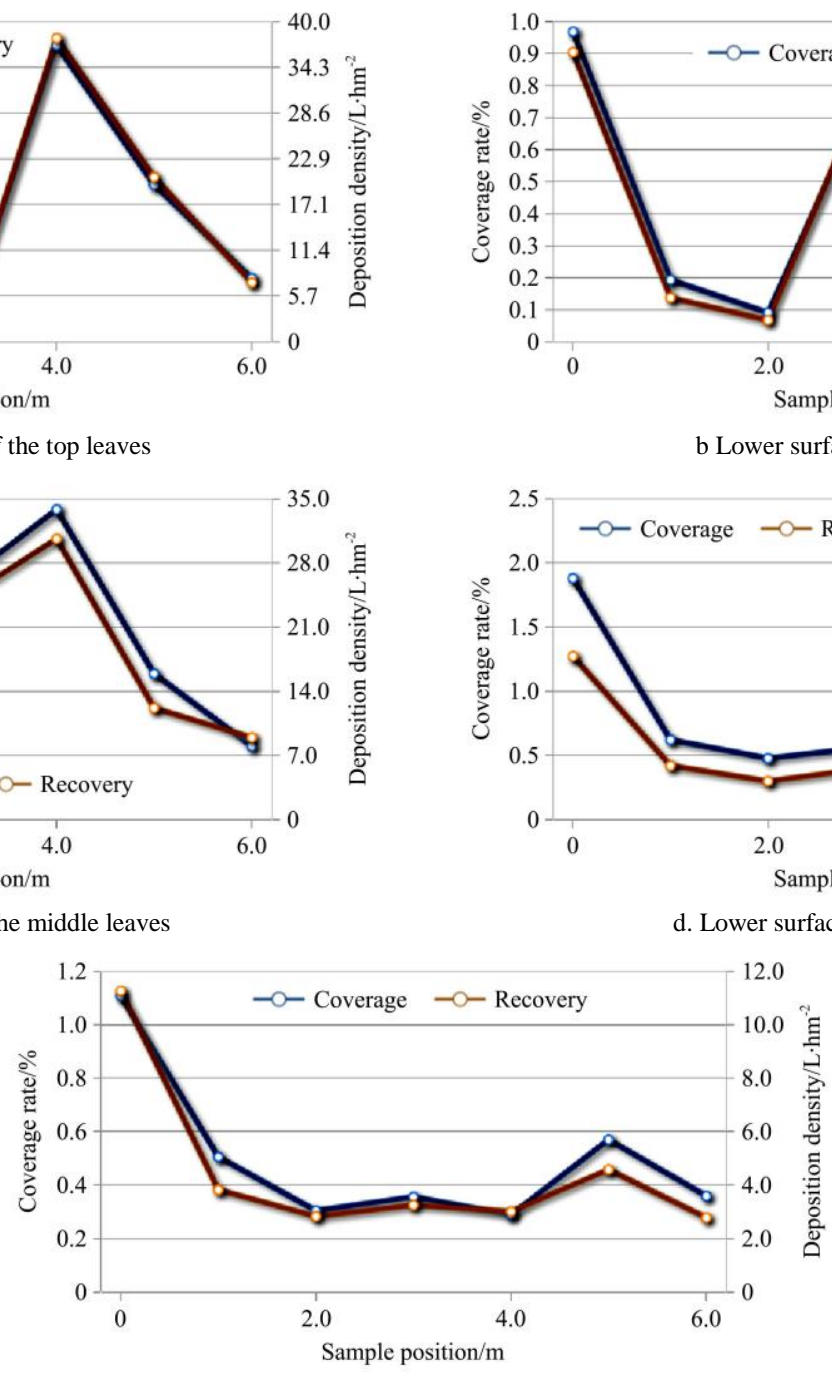

e. Bottom leaves

Figure 4 Coverage and recovery for the vertical positions on the rice plants

The distribution of droplet coverage was variable for different vertical positions. For the upper surfaces of the leaves at the top of the plants, the area directly below the nozzles had the highest coverage. For the lower surfaces of the leaves at the top of the plants, the best coverage was found in the area that the mist sprayed by two nozzles overlapped. For the upper surfaces of the leaves in the middle part of the plants, the best coverage was in the area within 2-4 $\mathrm{m}$ of the spraying width, and the best coverage for the lower surfaces of the leaves in the middle parts of the plants was in the area within $4-6 \mathrm{~m}$ of the spraying width. The droplet coverage for the leaves at the bottom of the plants was relatively low, and the coverage was less than $1 \%$ overall. The droplet coverage was higher for the leaves at the top of the plants than those in the middle and the bottom of the rice plants, as listed in Table 5.

Table 5 Distributions of droplet coverage and recovery along with vertical positions on rice plants

\begin{tabular}{lccc}
\hline \multicolumn{1}{c}{ Position } & $\begin{array}{c}\text { Overall } \\
\text { coverage/\% }\end{array}$ & $\begin{array}{c}\text { Average } \\
\text { coverage/\% }\end{array}$ & $\begin{array}{c}\text { Recovery } \\
/ \mathrm{L} \cdot \mathrm{hm}^{-2}\end{array}$ \\
\hline Upper surfaces of top leaves & 36.65 & 1.89 & 9.12 \\
Lower surfaces of top leaves & 21.73 & 1.13 & 5.07 \\
Upper surfaces of middle leaves & 17.36 & 0.81 & 3.26 \\
Lower surfaces of middle leaves & 16.19 & 0.75 & 1.14 \\
Bottom leaves & 11.34 & 0.41 & 5.10 \\
\hline
\end{tabular}

3.2 Analysis of the distribution of droplet size

Figure 5 shows the distribution of droplet size at different 
vertical positions on the rice plants. The ordinate indicates the droplet diameter and the density of deposition, and the abscissa indicates the actual length of the droplet collection belt. DV1, DV5, and DV9 refer to the droplet diameters when the volume accumulated
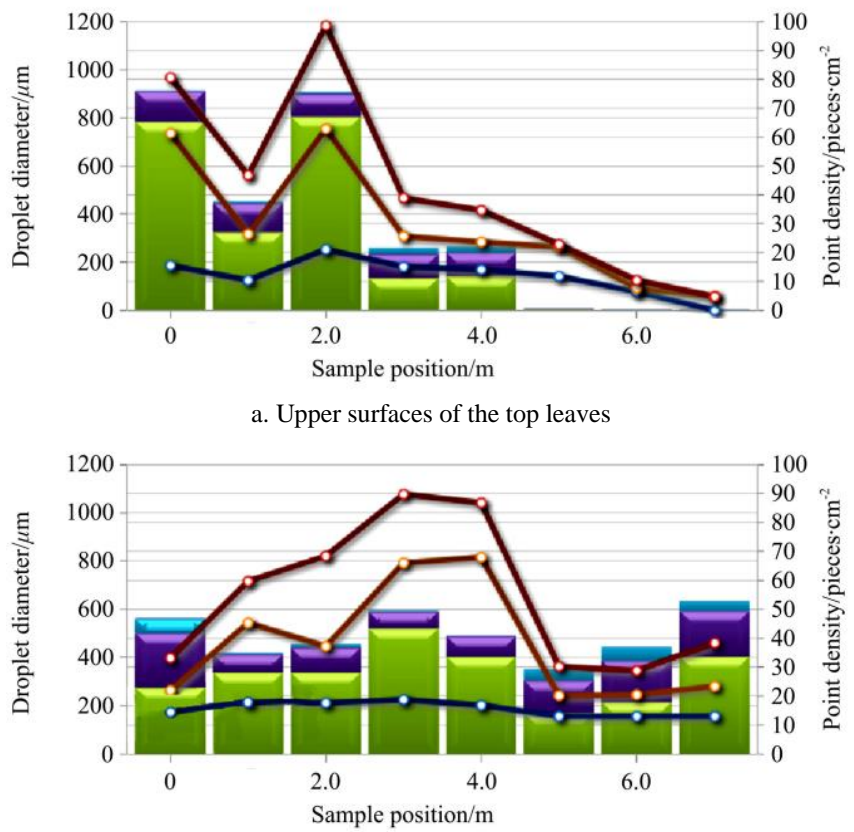

c. Upper surfaces of the middle leaves

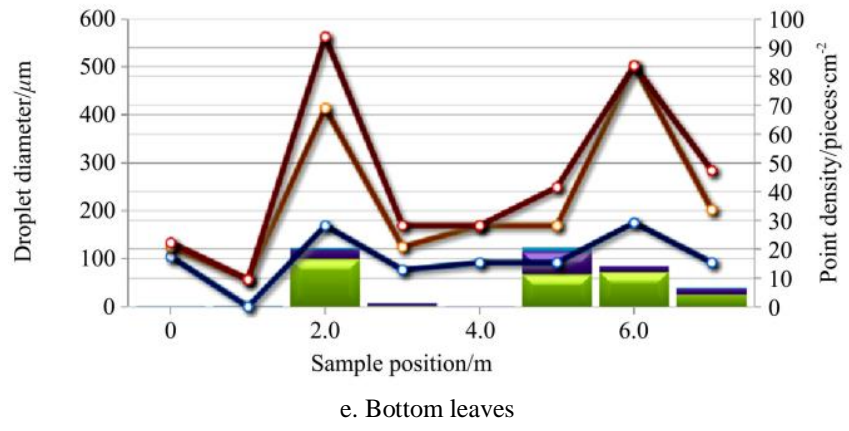

to $10 \%, 50 \%$, and $90 \%$, respectively. The diameter of the droplet was calculated from the deposition area and the diffusion coefficient and the number of droplets divided by the sampling area was the droplet density.

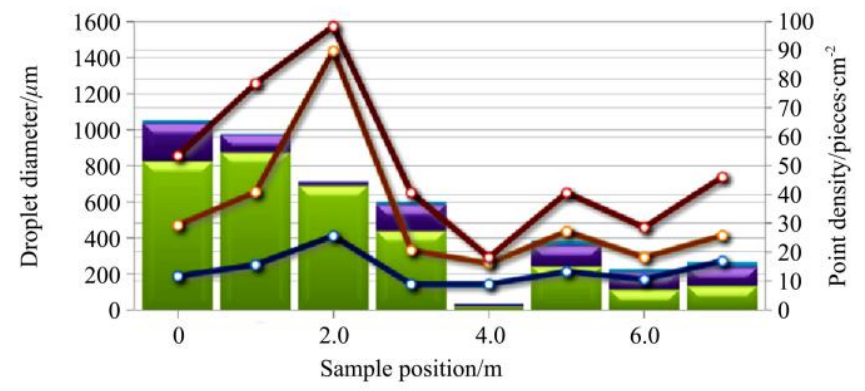

b Lower surfaces of the top leaves

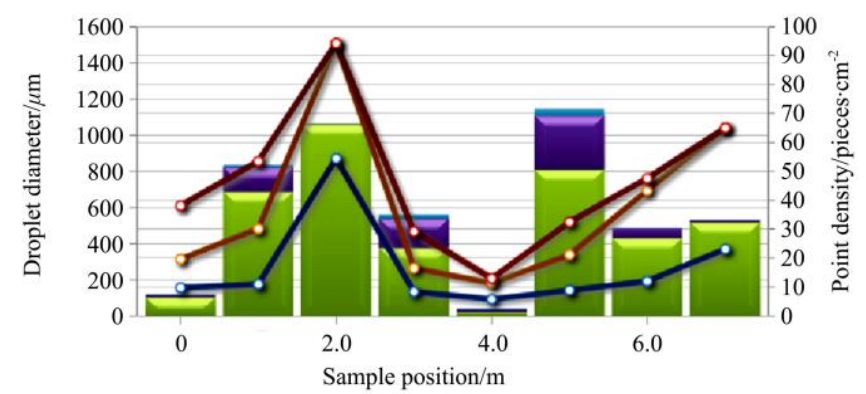

d. Lower surfaces of the middle leaves

$$
\begin{aligned}
& -\infty \text { Dv. } 1 \\
& -0-\text { Dv.5 } \\
& -0-\text { Dv. } 9 \\
& \sim 10 \% \text { dot density } \\
& 10 \% \sim 50 \% \text { dot density } \\
& 50 \% \sim 90 \% \text { dot density } \\
& 90 \% \sim \text { dot density }
\end{aligned}
$$

Figure 5 Distribution of droplet size for the vertical positions of the rice plants

During the plant protection UAV-based spraying, the liquids were dispersed by an atomizer. The droplets ejected from the nozzles varied in size, which was measured in micrometers. Droplet size is one of the important indicators for assessing the degree of atomization and the quality of the spraying operation. Himel and $\mathrm{Uk}^{[28,29]}$ proposed a theory of optimal droplet size in the mid-1970s. According to their theory, the droplet size that can be captured most easily by a living organism and can achieve the best control effect is called the optimal droplet size. The optimal droplet size may vary among insecticides, fungicides, and herbicides. For flying insects, the optimal droplet size is $10-50 \mu \mathrm{m}$, whereas the optimal size for the larvae of pests crawling on crop leaves is $30-150 \mu \mathrm{m}$. For plant diseases and weeds, the optimal sizes are 30-150 $\mu \mathrm{m}$ and 100-300 $\mu \mathrm{m}$, respectively. The results shown in Figure 5 indicate that the distribution of droplet size and droplet density significantly varied among different positions on the rice plants. The droplet density in the area below the rotors of the plant protection UAV was high, whereas the density was relatively low in the areas farther away from the rotors. Based on the regularity of distribution, the droplet size and density were clearly affected by the rotor wind field.

Table 6 shows the distribution of droplet size for different vertical positions on the rice plants. The highest droplet density was found at the top of the plants. The droplet density in the middle and at the bottom of the rice plants was similar. Based on the average value of droplet size, the size spectrum was $110-140 \mu \mathrm{m}$, which is suitable for the control of plant diseases and insect pests.

Table 6 Distribution of droplet size along with vertical positions on rice plants

\begin{tabular}{lccccc}
\hline \multicolumn{1}{c}{ Position } & $\begin{array}{c}\text { Droplet density } \\
\text { /droplet· } \mathrm{cm}^{2}\end{array}$ & $\begin{array}{c}\text { Average droplet } \\
\text { size/ } \mu \mathrm{m}\end{array}$ & $\begin{array}{c}\mathrm{DV} \mathrm{V}_{0.1} \\
/ \mu \mathrm{m}\end{array}$ & $\begin{array}{c}\mathrm{DV}_{0.5} \\
/ \mu \mathrm{m}\end{array}$ & $\begin{array}{c}\mathrm{DV}_{0.9} \\
/ \mu \mathrm{m}\end{array}$ \\
\hline $\begin{array}{l}\text { Upper surfaces of } \\
\text { top leaves }\end{array}$ & 32.4 & 129 & 144 & 202 & 498 \\
$\begin{array}{l}\text { Lower surfaces of } \\
\text { top leaves }\end{array}$ & 19.3 & 125 & 131 & 266 & 516 \\
$\begin{array}{l}\text { Upper surfaces of } \\
\text { middle leaves }\end{array}$ & 12.4 & 137 & 146 & 262 & 428 \\
$\begin{array}{l}\text { Lower surfaces of } \\
\text { middle leaves }\end{array}$ & 15.2 & 119 & 125 & 218 & 375 \\
\begin{tabular}{l} 
Bottom leaves \\
\hline
\end{tabular} & 19.0 & 111 & 133 & 312 & 598 \\
\hline
\end{tabular}

Note: $\mathrm{DV}_{0 . a}$ value is the droplet diameter $(\mu \mathrm{m})$ where $(a \times 100) \%$ of the spray volume is accumulated in droplets smaller than this value.

\subsection{Diffusion ratio and relative span}

The diffusion ratio (DR) and the relative span (RS) are internationally used indicators to assess the effect of spraying. The calculation of the two indicators was carried out with the following equations $^{[30,31]}$ : 
$\mathrm{DR}=$ volume median diameter/count median diameter

$$
\mathrm{RS}=\left(\mathrm{DV}_{0.9}-\mathrm{DV}_{0.1}\right) / \mathrm{DV}_{0.5}
$$

The ideal value of the droplet DR is 1 , which occurs if the volumes of all the droplets are the same. In general, if the range of DR is within 0.67-1, the sizes of the droplets are considered to be relatively uniform. For the value of RS, the smaller is more ideal,

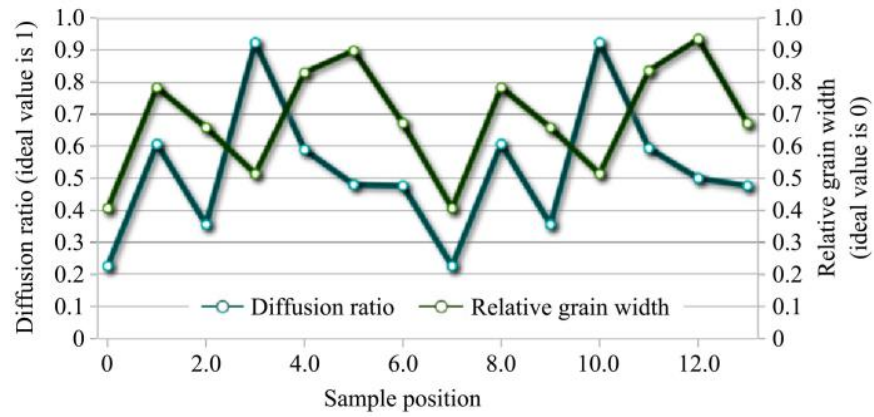

a. Upper surfaces of the top leaves

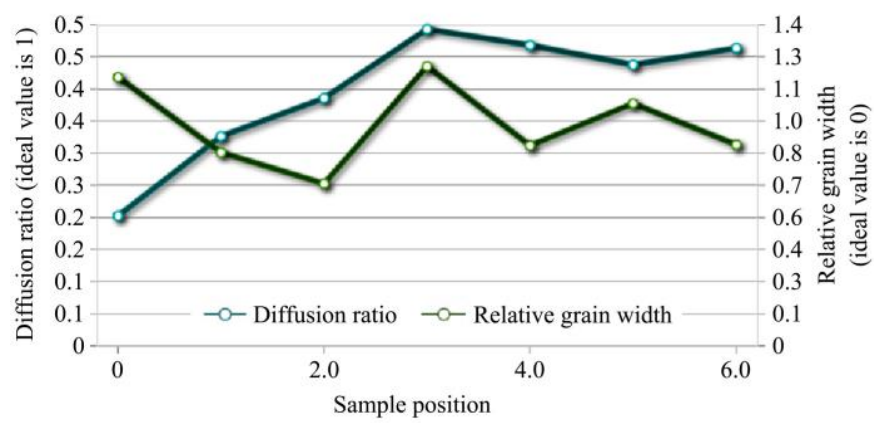

c. Upper surfaces of the middle leaves with the ideal value being 0 , which means that droplets with the same volume account for $80 \%$ of the total volume. Figure 6 shows that the DR and RS varied for different positions on the rice plants. Also, within the effective spray width of the UAVs, the distributions of the DR and RS show great variation.

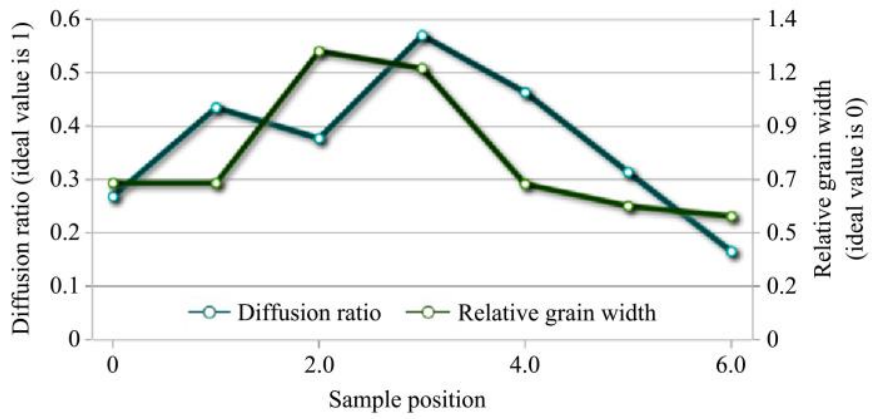

b Lower surfaces of the top leaves

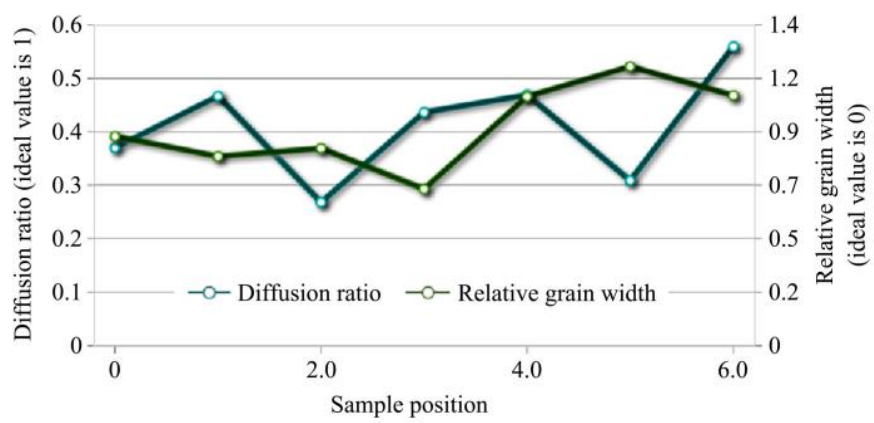

d. Lower surfaces of the middle leaves

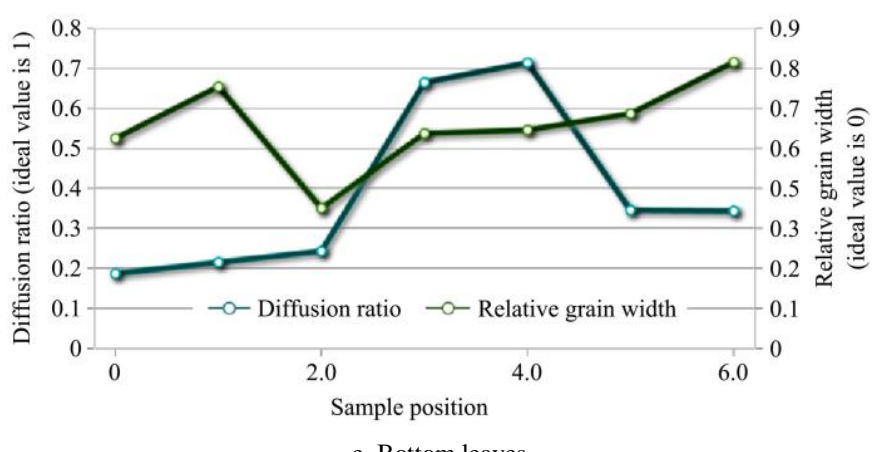

Figure 6 Diffusion ratio and relative span for the vertical positions of the rice plants

Table 7 shows the distributions of droplet DR and RS for different positions on the rice plants. The results indicate that for UAV-based spraying, the values of the DR and RS at the top of the plants were better than those in the middle and at the bottom of the rice plants. The lowest values of the DR and RS were found on the lower surfaces of the leaves in the middle part of the rice plants.

Table 7 Distributions of diffusion ratio and relative span for the positions in the vertical direction on rice plants

\begin{tabular}{lcc}
\hline \multicolumn{1}{c}{ Position } & Diffusion ratio & Relative span \\
\hline Upper surfaces of top leaves & 0.84 & 0.32 \\
Lower surfaces of top leaves & 0.57 & 0.44 \\
Upper surfaces of middle leaves & 0.52 & 0.75 \\
Lower surfaces of middle leaves & 0.46 & 0.97 \\
Bottom leaves & 0.37 & 0.78 \\
\hline
\end{tabular}

\section{Discussion}

In this study, an eight-rotor plant protection UAV was used to spray Japonica rice in a field in Northeast China. Water-sensitive spray cards were placed in different positions on the rice plant to acquire the data of the vertical distribution of the droplets sprayed by the UAV.

As shown in Figure 4, for the plant protection UAV-based spraying, the distribution of droplet coverage was poor. The best coverage was often in the area directly below the rotors, whereas the coverage in the areas beyond the rotors was poor. This might be due to the excessively small size of the droplets. Furthermore, the wind field of the rotors might play a key role in the deposition of the droplets. In the areas beyond the rotors, the forces and factors on the droplets, which may include the ambient wind field, boundary effect of the rotor wind field, and environmental temperature, were complex. Some of the droplets might have dissipated in the air before depositing on the leaves, while others might have drifted due to the complicated force of the wind. The distribution of the droplets on the upper and lower surfaces of the leaves was similar, which may be due to the upright shape of the Japonica rice in Northeast China and the effect of the wind field formed by multiple rotors. However, an in-depth investigation was not performed on 
the wind field of the rotors in this study. The data in Table 5 show that the droplet coverage was $58.38 \%, 33.55 \%$, and $11.34 \%$ for the top, middle, and bottom part of the rice plants, respectively, indicating that the droplets sprayed by the UAV could get through the canopy and deposit on the leaves in different positions on the plants. The data in Table 5 also show that the average droplet size in this study ranged between $110 \mu \mathrm{m}$ and $140 \mu \mathrm{m}$. The distributions of droplet size and droplet coverage were similar. The accumulation and overlapping of droplets might happen on the water-sensitive spray card. As a result, computer-based image analysis was adopted in this study. The droplet size estimated in this study may include similar errors described by other authors. The DR and RS were shown in Figure 6 and Table 7 indicate that the effects of plant protection UAV-based spraying were not ideal because the comprehensive index was lower than the reasonable level. At present, various kinds of plant protection UAVs have this problem, which may be caused by a combination of factors, including flight parameters, nozzles, and the specific features of crop plants. This study did not conduct an in-depth study on such factors.

Based on the results of this study, it is obvious that for plant protection UAV-based spraying, the distribution of droplet deposition is not only affected by the operating parameters of the UAV, but also significantly related to the wind field (rotor wind field and external wind field). The droplets sprayed by the UAV are prone to drifting in the air due to their small size. In order to determine the regularity of the droplet deposition of UAV-based spraying, it is necessary to study the regularity of the wind field distribution and the forces on the droplets. In this study, a type of software was used to distinguish droplet size. The actual deposition of droplets on the water-sensitive spray cards may be complicated. The number of droplets could be accurately identified if a small number of droplets were deposited on the card; however, if a large number of droplets were deposited on the card, overlap and adhesion of the droplets occurred. The image processing software employed in this research may have caused some errors in droplet analysis, but the overall results were reliable.

\section{Conclusions}

In this study, an electric eight-rotor plant protection UAV was used to spray a suspension containing tricyclazole and hexaconazole to study the droplet deposition along the vertical direction of rice plants growing in Northeast China. Based on the analysis of droplet deposition on the top, middle, and bottom leaves of rice plants, the following conclusions were drawn:

1) For plant protection UAV-based low-altitude spraying, the coverage of the droplets significantly varied for different positions on the rice plants. Within the effective spray width, the droplet coverage in the area below the rotors was the most comprehensive, whereas the coverage of the droplets was poor in the areas beyond the rotors. The overall coverage was $58.38 \%, 33.55 \%$, and $11.34 \%$ at the top, middle, and bottom of the rice plants, respectively.

2) For different vertical positions on the rice plants, the average droplet size ranged from $110-140 \mu \mathrm{m}$, which is suitable for the control of plant diseases and insect pests. At the top of the plants, the droplet density was the highest, and in the middle and at the bottom of the rice plants, the distributions of droplet density were similar.

3) The droplet diffusion ratio (0.84) at the top of the rice plants was better than that in the middle (0.57) and at the bottom (0.37) of the plants. The RS of the spray could meet the requirements for the actual application. Except for the middle part of the plants, the $\mathrm{RS}$ at the top and bottom of the plants was higher than the standard value of 0.67 .

\section{Acknowledgements}

The authors gratefully acknowledge that this work was financially supported by the Key R\&D Program of Liaoning Province (2020JH2/10200038) and the National Key R\&D Program of China (2016YFD0200700).

\section{[References]}

[1] Zhang Y L, Lan Y B, Fritz B K, Xue X Y. Development of aerial electrostatic spraying systems in the United States and applications in China. Transactions of the CSAE, 2016; 32 (10): 1-7. (in Chinese)

[2] Ru Y, Jin L, Zhou H P, Jia Z C. Performance experiment of rotary hydraulic atomizing nozzle for aerial spraying application. Transactions of the CSAE, 2014; 30(3): 50-55. (in Chinese)

[3] Wang C L, He X K, Wang X N, Bonds J, Herbst A, Wang Z G, et al. Testing method of spatial pesticide spraying deposition quality balance for unmanned aerial vehicle. Transactions of the CSAE, 2016; 32(11): 54-61. (in Chinese)

[4] Yao W X, Lan Y B, Hoffmann W C, Li J Y, Guo S, Zhang H H, et al. Droplet size distribution characteristics of aerial nozzles by Bell206L4 helicopter under medium and low airflow velocity wind tunnel condition and field verification test. Applied Sciences, 2020; 10(6): 2179. doi: 10.3390/app10062179.

[5] Xue X Y, Lan Y B. Agricultural aviation applications in USA. Transactions of the CSAM, 2013; 44(5): 194-201. (in Chinese)

[6] Martin D E, Carlton J B. Air speed and orifice size affect spray droplet spectra from an aerial electrostatic nozzle for rotary-wing applications. Atomization and sprays, 2012; 22(12): 997-1010.

[7] Fritz B K, Hoffmann W C. Establishing reference nozzles for classification of aerial application spray technologies. Int. J. Precis. Agric. Aviat., 2018; 1: 10-14.

[8] Yao W X, Lan Y B, Wen S, Zhang H H, Zhang Y L, Wang J, et al. Evaluation of droplet deposition and effect of variable-rate application by a manned helicopter with AG-NAV Guía system. Int J Agric \& Biol Eng, 2019; 12(1): 172-178.

[9] Lan Y B, Thomson S J, Huang Y, Hoffmann W C, Zhang H H. Current status and future directions of precision aerial application for site-specific crop management in the USA. Computers \& Electronics in Agriculture, 2010; 74(1): 34-38

[10] Yin X C, Lan Y B, Wen S, Deng J Z, Zhang J L, Zhang J T. The development of Japan agricultural aviation technology and its enlightenment for China. Journal of South China Agricultural University, 2018; 39(2): 1-8. (in Chinese)

[11] Lan Y B, Chen S D, Fritz B K. Current status and future trends of precision agricultural aviation technologies. Int J Agric \& Biol Eng, 2017; 10(3): 1-17.

[12] Du W, Cao Y L, Xu T Y, Cong L, Hong X, Tang R. Effect and assessment of UAV spraying parameters at japonica rice canopies. Journal of Agricultural Mechanization Research, 2017; 4: 182-186, 191. (in Chinese)

[13] Zhang S C, Xue X Y, Qin W C, Sun Z, Ding S M, Zhou L X. Simulation and experimental verification of aerial spraying drift on N-3 unmanned spraying helicopter. Transactions of the CSAE, 2015; 31(3): 87-93. (in Chinese)

[14] Qiu B J, Wang L W, Cai D L, Wu J H, Ding G R, Guan X P. Effects of flight altitude and speed of unmanned helicopter on spray deposition uniform. Transactions of the CSAE, 2013; 29(24): 25-32. (in Chinese)

[15] Giles D, Billing R. Deployment and performance of a UAV for crop spraying. Chemical Engineering Transactions, 2015; 44: 307-312.

[16] Yuan H Z, Wang G B. Effects of droplet size and deposition density on field efficacy of pesticides. Plant Protection, 2015; 41(6): 9-16. (in Chinese)

[17] Martinez-Guanter J, Agüera P, Agüera J, Pérez-Ruiz M. Spray and economics assessment of a UAV-based ultra-low-volume application in olive and citrus orchards. Precision Agriculture, 2020; 21: 226-243.

[18] Berner B, Chojnacki J. Influence of the air stream produced by the drone on the sedimentation of the sprayed liquid that contains entomopathogenic 
nematodes. Journal of Research and Applications in Agricultural Engineering, 2017; 62(3): 26-29.

[19] Teske M, Wachspress D, Thistle H. The prediction of aerial spray release from UAVs. Transactions of the ASABE, 2018; 61(3): 909-918.

[20] Chen S D, Lan Y B, Li J Y, Zhou Z Y, Jin J, Liu A M. Effect of spray parameters of small unmanned helicopter on distribution regularity of droplet deposition in hybrid rice canopy. Transactions of the CSAE, 2016; 32(17): 40-46. (in Chinese).

[21] Ru Y, Zhu C Y, Bao R. Spray drift model of droplets and analysis of influencing factors based on wind tunnel. Transactions of the Chinese Society for Agricultural Machinery, 2014; 45(10): 66-72. (in Chinese)

[22] Fritz B K, Hoffmann W C, Bagley W E, Kruger G R, Czaczyk Z, Henry R S Influence of air shear and adjuvants on spray atomization. ASTM International, 2014; 33: 151-173

[23] Qin W C, Xue X Y, Zhou L X, Zhang S C, Sun Z, Kong W, et al. Effects of spraying parameters of unmanned aerial vehicle on droplets deposition distribution of maize canopies. Transactions of the CSAE, 2014; 30(5): 50-56. (in Chinese)

[24] Li J Y, Zhou Z Y, Lan Y B, Hu L, Zang Y, Liu A M, et al. Distribution of canopy wind field produced by rotor unmanned aerial vehicle pollination operation. Transactions of the CSAE, 2015; 31(3): 77-86. (in Chinese)
[25] Sudheer K P, Panda R K. Digital image processing for determining drop sizes from irrigation spray nozzles. Agricultural Water Management, 2000; 45(2): 159-167.

[26] Yao W X, Guo S, Yu F H, Du W, Meng Y H, Wang J, et al. Droplet deposition and spatial drift distribution characteristics of aerial spraying based on the determination of effective swath. Int J Precis Agric Aviat, 2021; 4(1): 36-43.

[27] Hilz E, Vermeer A W P. Spray drift review: The extent to which a formulation can contribute to spray drift reduction. Crop Protection, 2013 44(1): 75-83.

[28] Himel C M. The optimum size for insecticide spray droplets ${ }^{1,2}$. Journal of Economic Entomology, 1969; 62(4): 919-925.

[29] Uk S. Tracing insecticide spray droplets by sizes on natural surfaces. the state of the art and its value. Pesticide Science, 1977; 8(5): 501-509.

[30] Guo S, Li J Y, Yao W X, Zhan Y L, Li Y F, Shi Y Y. Distribution characteristics on droplet deposition of wind field vortex formed by multirotor UAV. PLoS ONE, 2019; 14(7): e0220024. doi: 10.1371/ journal.pone.0220024.

[31] Thomson S J, Smith L A, Hanks J E. Evaluation of application accuracy and performance of a hydraulically operated variable-rate aerial application system. Transactions of the ASABE, 2009; 52(3): 715-722. 